Bull. Egypt. Soc. Physiol. Sci. Vol. (41) tissue (3), 316-330

\author{
Bull. of Egyp. Soc. Physiol. Sci. \\ (Official Journal of Egyptian Society for Physiological Sciences) \\ (pISSN: 1110-0842; eISSN: 2356-9514)
}

\title{
Role of Renin Angiotensin System in Isoproterenol-Induced Myocardial Infarction in
}

\author{
Male Rats \\ Lashin S. Lashin ${ }^{1,2}$, Ahmed M. shata ${ }^{3,4}$, Manal A. Hamouda ${ }^{4}$, Mona G. Elhadidy ${ }^{1}$ \\ ${ }^{1}$ Physiology Department, Faculty of Medicine, Mansoura, University, Mansoura, Egypt \\ 2Physiology Department, Faculty of Physical Therapy, Horus University, New Damietta, Egypt \\ 3Clinical pharmacology department, Faculty of Medicine, Mansoura university, Egypt. \\ ${ }^{4}$ Pharmacy Practice Department, Faculty of Pharmacy, Delta University for Science and Technology, Gamasa, \\ Egypt
}

Submit Date: Sept 25, 2020

Accept Date: Oct 27, 2020

Available Online: Feb 1, 2021

\section{Keywords}

- Myocardial

infarction

ISO

ACEIs

ARBs

HO-1

Nrf2

oxidative stress

\begin{abstract}
Background: Myocardial infarction (MI) is a necrosis of cardiomyocytes due to prolonged myocardial ischemia, the injured heart characterized by activation of the reninangiotensin system (RAS). The cardiac effect of enalapril and losartan was determined in Isoproterenol (ISO) induced MI rat model. Methods: Twenty-four adult male rats were randomly divided into four groups of six rats each, Group-I- Normal rats, group II-ISO (150 mg/kg once daily for two successive days), Group-III-Enalapril pretreated, and Group-IV-Losartan pretreated rats. Enalapril and losartan were given by gavage at dose $10 \mathrm{mg} / \mathrm{kg}$ and $30 \mathrm{mg} / \mathrm{kg}$, respectively the treatment started 3 days before ISO injection and continue for 5 days after 2 nd ISO dose. Results: ISO-induced group showed significant increase in the cardiac enzymes lactate dehydrogenase (LDH) and creatine kinase-MB (CK-MB), accompanied with marked decrease in the catalase (CAT) and glutathione (GSH) and Bcl2. Both enalapril and losartan induce significant reduction of cardiac enzymes and systolic blood pressure and improve oxidative stress which confirmed by reduction of Malondialdehyde (MDA) and elevation of antioxidant enzymes and markedly upregulated the expression of Bcl2, Nrf-2 and HO-1. Moreover, Both enalapril and losartan reversed the histopathological changes induce by ISO and thus exhibits its cardioprotective activity. Moreover, treated rats partially minimize myocardial necrosis and interstitial edema. In conclusion: Enalapril and losartan ameliorates oxidative stress, inhibit apoptosis and improve cardiac dysfunction by alteration of Nrf2/HO-1 signaling pathway. It also reduces cardiac enzymes and improves blood pressure without affection of serum $\mathrm{K}$ on short term therapy in MI.
\end{abstract}

Corresponding author: Mona G. Elhadidy, Department of Medical Physiology, Faculty of Medicine, 24 Gomhouria St., Mansoura, Egypt. 35516. Email: mona.gaber2012@yahoo.com. Tel; 00201001331750 


\section{INTRODUCTION}

Cardiovascular diseases have been considered as the most common cause of death worldwide and in developed countries during the past century; however, now, there is an increase in cardiovascular disease rates in low- and middleincome countries. Coronary heart diseases account for $10 \%$ to $35 \%$ of deaths. One of these diseases is MI which occurs due to the prolonged myocardium ischemia that end with necrosis of myocytes (1). Cardiac arrhythmias in an injured myocardium also can result due to reactive oxygen species (ROS), ion channels alteration in cell membranes and abnormal gap junction channels (2). The renin-angiotensin-aldosterone system (RAAS) has a significant role in a diversity of clinical settings, like MI, atherosclerosis, hypertension, left ventricular hypertrophy and heart failure. As a consequence, the RAAS characterizes a rational beneficial area in the controlling of hypertension, renal disease, and cardiovascular disease (2). There is an effort to improve the acute management of MI through life saving interventions such as drug therapies, percutaneous coronary interventions and strategies to reducing deaths caused by cardiovascular diseases.

Renin angiotensin system antagonizing drugs such as angiotensin-converting enzyme inhibitors (ACEIs), angiotensin receptor blockers (ARBs), and mineralocorticoid receptor antagonists (MRAs) have become a most effective antihypertensive drugs (3). Telmisartan one of ARBs induced marked elevation in the angiotensin converting enzyme-2 (ACE-2) gene expressions in the myocardium of pressure-overloaded rats and also improves hypertrophy of vessels in spontaneously hypertensive rats by changing the expression of ACE-2 with an obvious reverse of extracellular-signal regulated kinase 1/2 (ERK1/2) and c- Jun N-terminal kinase (JNK) phosphorylation signaling pathways. These results conclude that angiotensin (1-7) causes vascular dilatation, which antagonizes the vasoconstriction mediated by angiotensin II type 1 receptor (AT1R), thus result in lowering of blood pressure, decrease of cardiac hypertrophy, fibrosis and renal problems (4).

Heme oxygenase-1 (HO-1) is the ratelimiting enzyme which mediates the degradation of heme to biliverdin through a reaction that produces carbon monoxide and releases iron (5). HO-1 is strongly elevated by exposure to oxidative stress and shows cytoprotective effects via the anti-proliferative, anti-inflammatory, and antiapoptotic actions (6). nuclear factor erythroid 2related factor 2 (Nrf2) a key transcription factor, plays an important role in the synthesis of endogenous antioxidant enzymes against oxidative stress. Several reports have demonstrated that Nrf2 protects cardiomyocytes against oxidative stress through increasing detoxification pathways and antioxidant potentials $(7,8)$. Several studies demonstrated that ISO induce overproduction of free radicals which modulate redox signaling pathway like Nrf2/ HO-1 to protect myocardium from induced damage or injury which mediated by enhancing oxidative status $(9,10)$. Our present work evaluated the role of RAS in MI induced by isoproterenol and effects of its block by ACEIs and ARBs on progression of MI. Moreover, we compared between two types of treatment and evaluate their efficacy in control of MI via altering 
Nrf2/HO-1 redox signaling pathway.

\section{Materials and methods}

Experimental animals: Data will be attained from laboratory-based studies by adult Male Sprague Dawley rats aged 3 to 4 months and weighing nearly $150-180 \mathrm{~g}$ from medical experimental research center (MERC), Faculty of Medicine, Mansoura University, Egypt. Rats were kept in polypropylene cages, acclimated at standard environmental conditions of $25 \pm 2^{\circ} \mathrm{C}, 50 \pm 15 \%$ humidity, and a natural light-dark cycle having free access to standard pellet diet and tap water ad libitum. This study was carried out in agreement with the national and international guidelines for the care and use of animals. Our Local Committee of Animal Care and Used approved this protocol (Code number R.19.09.630).

Drugs and chemicals: Isoproterenol hydrochloride (ISO) was purchased from Sigma-Aldrich (St. Louis, USA), Losartan (Merk Sharp and Dohme, UK) and enalapril tablets (Mulit-Apex for pharmaceutical industries, Egypt). Both losartan and enalapril were dissolved in distilled water.

Induction of ISO-induced MI in rat model: ISO is non-specific $\beta$-adrenergic receptors agonizing drugs that cause marked stressful activation in myocardium that lead to necrosis in the cardiac muscles and depletion of its energy reserve stores. Irreversible cellular damage and necrosis result from a complex biochemical and structural changes. Rats were received ISO $(150 \mathrm{mg} / \mathrm{kg})$ intraperitoneally for 2 consecutive days with 24 hours intervals (11).

Experimental protocol: Twenty-four adult male Sprague Dawley rats will be divided into 4 groups (6 rats each):
1. Group I- Normal rats were intraperitoneally injected with saline

2. Group II- Isoprenaline group received ISO $150 \mathrm{mg} / \mathrm{Kg}$ twice with $24 \mathrm{hrs}$ interval

3. Group III- Enalapril (10 $\mathrm{mg} / \mathrm{kg}$, orally starting 3 days before ISO injection and continue for 5 days after 2 nd ISO dose) (12).

4. Group IV- Losartan (30 mg/kg, orally starting 3 days before ISO injection and continue for 5 days after 2nd ISO dose)(13).

Blood pressure measurement: Systolic blood pressure (SBP) of all experimental animals was measured before induction of MI (basal) and at 3, and 5 days after induction of MI. This blood pressure was measured in Physiology Department, Faculty of Medicine, Mansoura, University, Mansoura, Egypt by blood pressure monitor (ML 125 NIBP, AD Instruments, Australia) from the tail of conscious rats using the tail-cuff technique. The average of at least three measurements of blood pressure was calculated each occasion.

\section{Collection of blood samples and harvesting heart:}

Blood samples were obtained from ophthalmic veins from all groups with halothane light anesthesia before induction of MI (basal) and at 3, and 5 days after induction of MI. From collected blood sample, the serum was separated by centrifuging the blood at 3500 for $10 \mathrm{~min}$, for estimating cardiac enzymes (CK-MB and $\mathrm{LDH}$ ) and potassium $(\mathrm{K})$ levels. At the end of the experiment, rats were sacrificed with a high dose of sodium thiopental $(120 \mathrm{mg} / \mathrm{Kg})$ and the chest was opened and the heart was rapidly removed. Later, the cardiac muscle was dissected into 3 parts; the small part weighted and preserved for determination of oxidative stress markers. The second part was placed in RNAlater Stabilization 
Solution (ThermoFisher Scientific) at $4^{\circ} \mathrm{C}$ overnight before being stored at $-80^{\circ} \mathrm{C}$ until isolation of total RNA and subsequent RT-PCR meanwhile, the last part was preserved in $10 \%$ neutral formalin for subsequent histological examination.

Assay of the cardiac enzymes: Using commercial kits according to labeled instructions, cardiac enzymes CK-MB (bioMe'rieux Diagnostics, Milan, Italy) and LDH (Bayer Diagnostics Ltd., Baroda, India), serum K (bioMe'rieux Diagnostics, Milan, Italy) were measured.

Estimation of oxidative stress markers: Cardiac homogenate was used to assess the levels of Malondialdehyde (MDA), catalase (CAT) and glutathione (GSH) by colorimetric kits brought from Bio-Diagnostics, Giza, Egypt according to labeled instructions.

Detection of mRNA of Bcl2, Nrf2 and HO-1 by RT-PCR in cardiac tissues: Total RNA was extracted from cardiac tissue and purity and concentration was assessed. From the RNA template; cDNA was synthesized by reverse transcription. All the manipulations were carried out according to the kit instructions. The corresponding primers (Table I) were added, and the expression of Bcl2, Nrf2 and HO-1 genes was assessed by RT-PCR. GAPDH was used as the internal reference. The procedures of RNA extraction and cDNA synthesis were done in Nile Center for Experimental Research, Mansoura, Egypt.

Table (I): RT-PCR primer sequences:

\begin{tabular}{|l|l|}
\hline \multicolumn{1}{|c|}{ Gene name } & \multicolumn{1}{c|}{ Primer sequences } \\
\hline Bcl-2 & $\begin{array}{l}\text { Forward: 5'-TGAACCGGCATCTGCACAC-3' } \\
\text { Reverse: 5'-CGTCTTCAGAGACAGCCAGGAG-3' }\end{array}$ \\
\hline Nrf2 & $\begin{array}{l}\text { Forward: 5'-CATTTGTAGATGACCATGAGTCGC-3' } \\
\text { Reverse: 5'-GCTCCATGTCCTGCTGTATGC-3' }\end{array}$ \\
\hline HO-1 & $\begin{array}{l}\text { Forward: 5'-TCACCTTCCCGAGCATCGAC-3' } \\
\text { Reverse: 5'-TCACCCTGTGCTTGACCTCG-3' }\end{array}$ \\
\hline GAPDH & $\begin{array}{l}\text { Forward: 5'-ATGGCACCGTCAAGGCTGAG-3' } \\
\text { Reverse: 5'-TGTCAGGTACGGTAGTGACG-3' }\end{array}$ \\
\hline
\end{tabular}

Histopathological examination: The part of the heart fixed in formalin was processed in paraffin blocked then slides were processed and stained with hematoxylin and eosin (H\&E). An expert pathologist examined the slides for myocardial cell necrosis, interstitial edema and inflammatory cells infiltrates via light microscope. The degree of myocardial damage was scored as follow: no changes; + or mild damage $=$ focal myocyte damage or little multifocal degeneration with slight degree of inflammatory process; ++ or moderate damage =extensive myofibrillary degeneration and/or diffuse inflammatory process and +++ or marked $=$ necrosis with obvious diffuse inflammatory process. All studied rats were subdivided into 3 groups: group A (absent histopathological change), group B (little 
histopathological change) and group $\mathrm{C}$ (moderate

Statistical analysis: Statistical analysis was done using SPSS 22.0 (SPSS, Inc., Chicago, IL, USA). The statistical significance was done by one-way analysis of variance (ANOVA) followed by Dunnet's comparison test. The values were expressed as mean \pm SEM and $p<0.05$ was considered significant.

\section{Results}

Figure 1 revealed that there was a significant reduction of systolic blood pressure when comparing enalapril treated group $(\mathrm{p}<0.002)$ and losartan treated group $(\mathrm{p}<0.006)$ with ISO group in basal conditions but this effect is not evident after 3 or 5 days. Also, this effect is evident when comparing both pretreated group ( $p$ $<0.001$ ) with negative control rats.

Administration of ISO resulted in marked decrease in the level of both GSH and catalase with significant increase in the level of MDA when compared with normal rats. On the other hand, losartan improve significantly antioxidant status through elevation of GSH $(p<0.001)$ and increase of catalase $(p<0.001)$ and reduce significantly lipid peroxiding marker (MDA) $(p<0.004)$ when comparing to positive control group. Meanwhile, these changes are not evident with enalapril as belong the three above parameters Table 1. and/ or marked histopathological changes).

In Figure 2 administration of ISO showed a significant increase in the concentrations of cardiac enzymes $\mathrm{CK}-\mathrm{MB}$ in isoprenaline group when compared with control negative group. Both enalapril and losartan induce significant reduction of this cardiac enzyme which was significant increase in ISO group after 3 days $(\mathrm{p}<0.001, \mathrm{p}$ $<0.001$ respectively) and 5 days $(\mathrm{p}<0.001, \mathrm{p}$ $<0.001$ respectively).

Also, in Figure 3 a significant increase in the level of LDH was noticed in rats treated with ISO when compared to control rats. Pretreatment of rats with enalapril and losartan induce significant reduction of LDH after 3 days $(p<$ $0.001, \mathrm{p}<0.001$ respectively) and 5 days $(\mathrm{p}<$ $0.001, \mathrm{p}<0.001$ respectively). However, these effects on cardiac enzymes were not evident initially at start of therapy.

The serum level of $\mathrm{K}$ was measured for rats of all experimental groups as it demonstrated in Figure 4, all medication doesn't produce any significant changes in serum $\mathrm{K}$ either initially or after 3 or 5 days of medications.

Table (1): Effect of enalapril and losartan on GSH, Catalase and MDA:

\begin{tabular}{|c|c|c|c|}
\hline & $\begin{array}{c}\text { GSH } \\
\text { (mg/g tissue) }\end{array}$ & $\begin{array}{c}\text { Catalase } \\
\text { (U/g tissue) }\end{array}$ & $\begin{array}{c}\text { MDA } \\
\text { (nmol/g tissue) }\end{array}$ \\
\hline Normal group & $222.73 \pm 5.2$ & $17.87 \pm 0.91$ & $2.02 \pm 0.06$ \\
\hline ISO group & $19.94 \pm 1.4^{p 2}$ & $4.2 \pm 0.3^{p 2}$ & $18.6 \pm 1.6^{p 2}$ \\
\hline Enalapril treated group & $31.27 \pm 2.6^{\mathrm{p} 2}$ & $4.1 \pm 0.12^{\mathrm{p} 2}$ & $19.98 \pm 2.1^{p^{2}}$ \\
\hline Losartan treated group & $76.62 \pm 2.5^{p 1, p 2}$ & $7.7 \pm 0.57^{p 1, p 2}$ & $10.71 \pm 1.02^{p 1, p 2}$ \\
\hline
\end{tabular}

Test used: One-way ANOVA followed by Dunnet comparison test. Values expressed as means \pm SEM.$^{\mathrm{P}}$ significance vs isoprenaline group ${ }^{\mathrm{P} 2}$ significance vs negative group. 


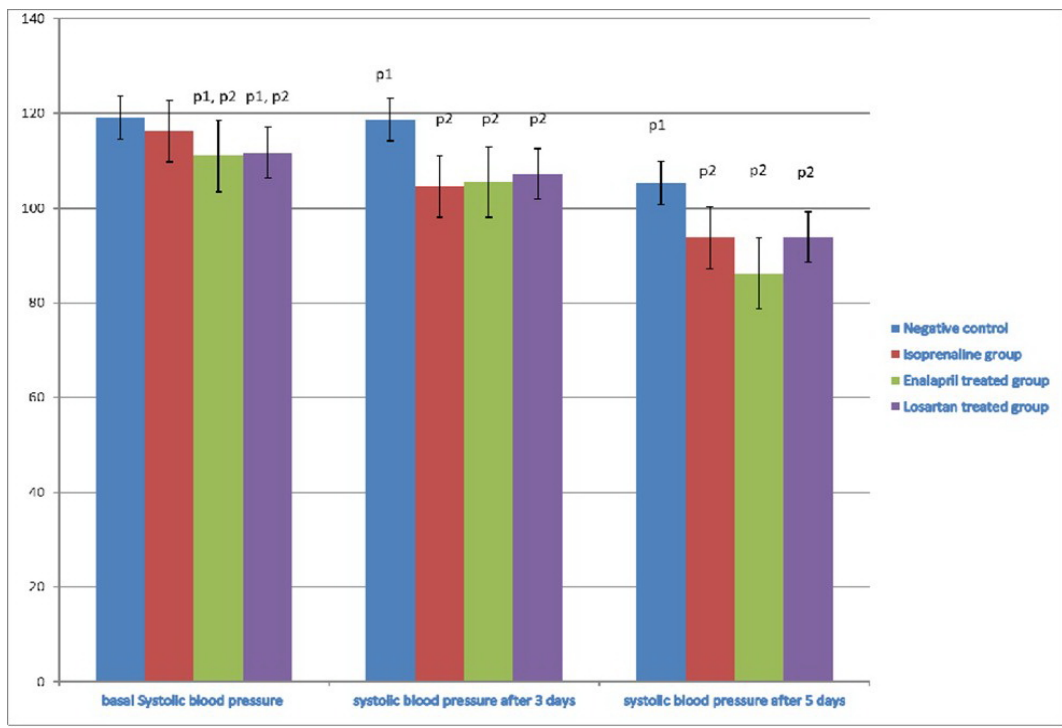

Figure (1): Effect of enalapril and losartan on systolic blood pressure (mmHg). Test used: One-way ANOVA followed by Dunnet comparison test. Values expressed as means \pm SEM$^{\mathrm{P} 1}$ significance vs isoprenaline group, ${ }^{\mathrm{P} 2}$ significance vs negative group.

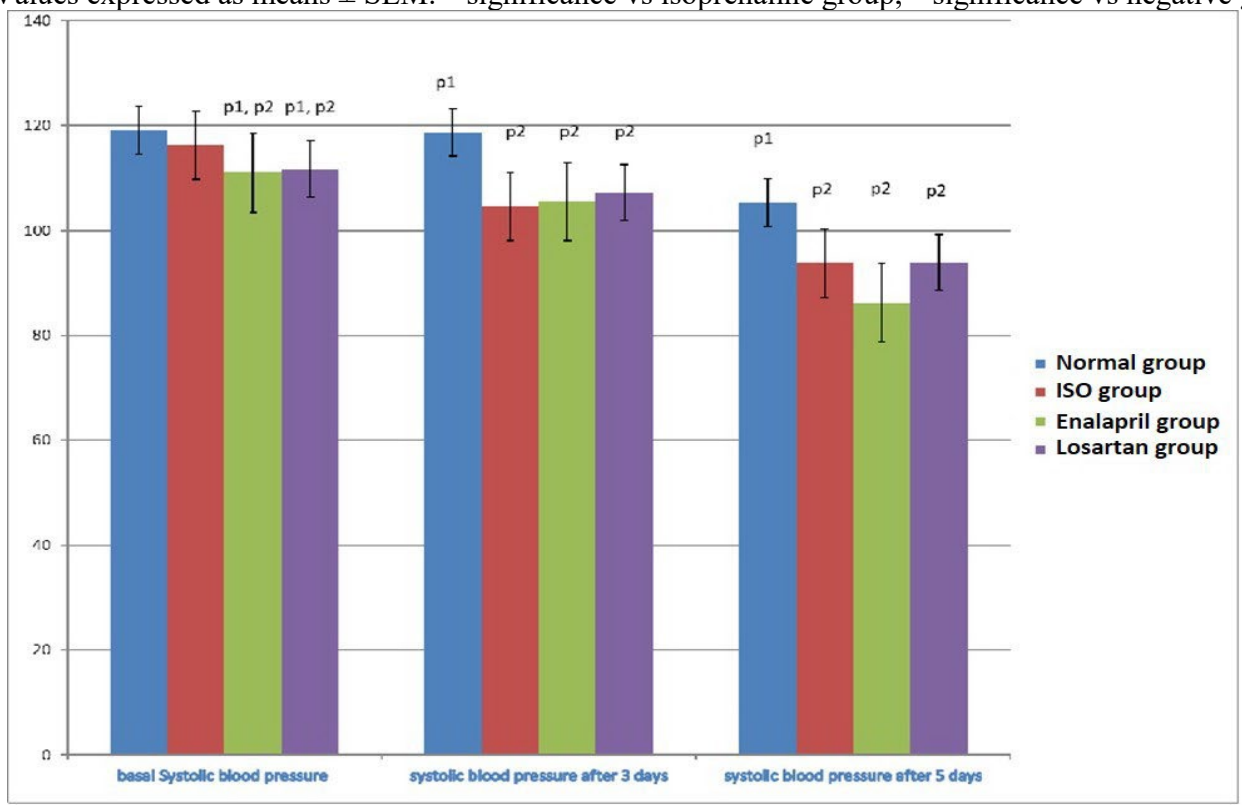

Figure (2): Effect of enalapril and losartan on CK-MB (U/L). Test used: One-way ANOVA followed by Dunnet comparison test. Test used: Values expressed as means \pm SEM. ${ }^{\mathrm{P} 1}$ significance vs isoprenaline group, ${ }^{\mathrm{P} 2}$ significance vs negative group.

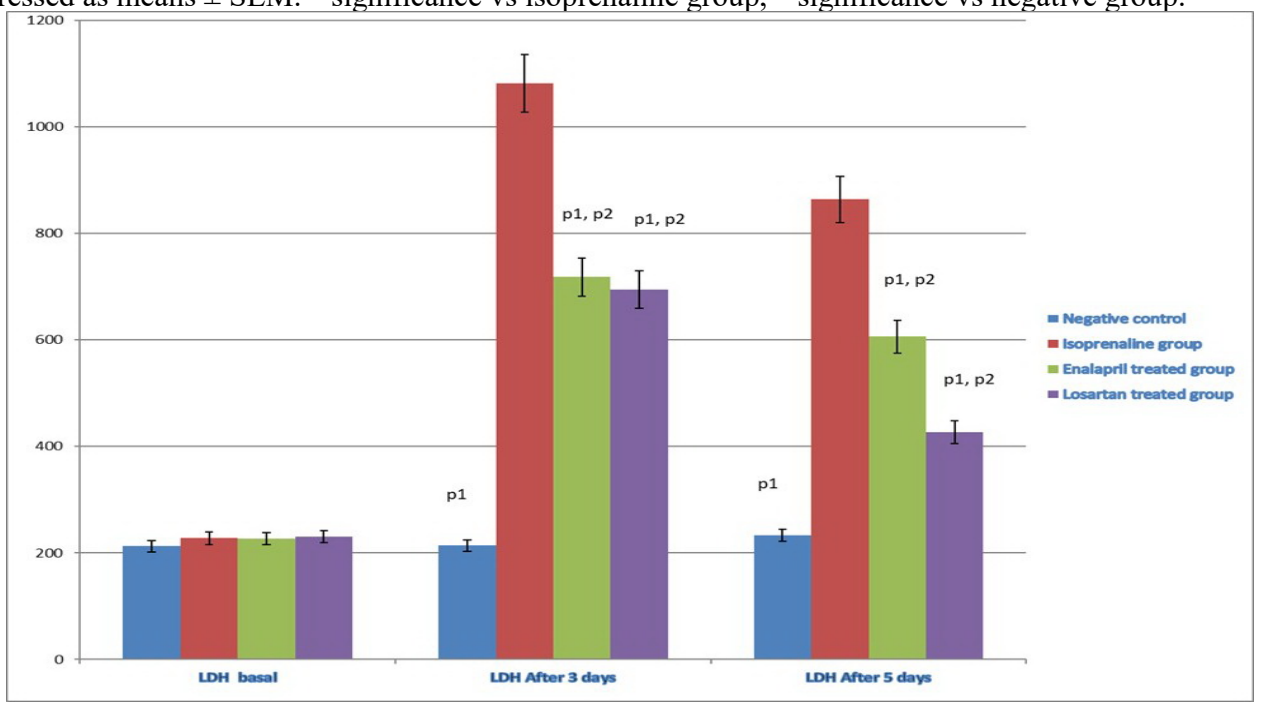

Figure (3): Effect of enalapril and losartan on LDH (U/L). Test used: One-way ANOVA followed by Dunnet comparison test. Values expressed as means \pm SEM. ${ }^{\mathrm{P} 1}$ significance vs isoprenaline group, ${ }^{\mathrm{P} 2}$ significance vs negative group. 


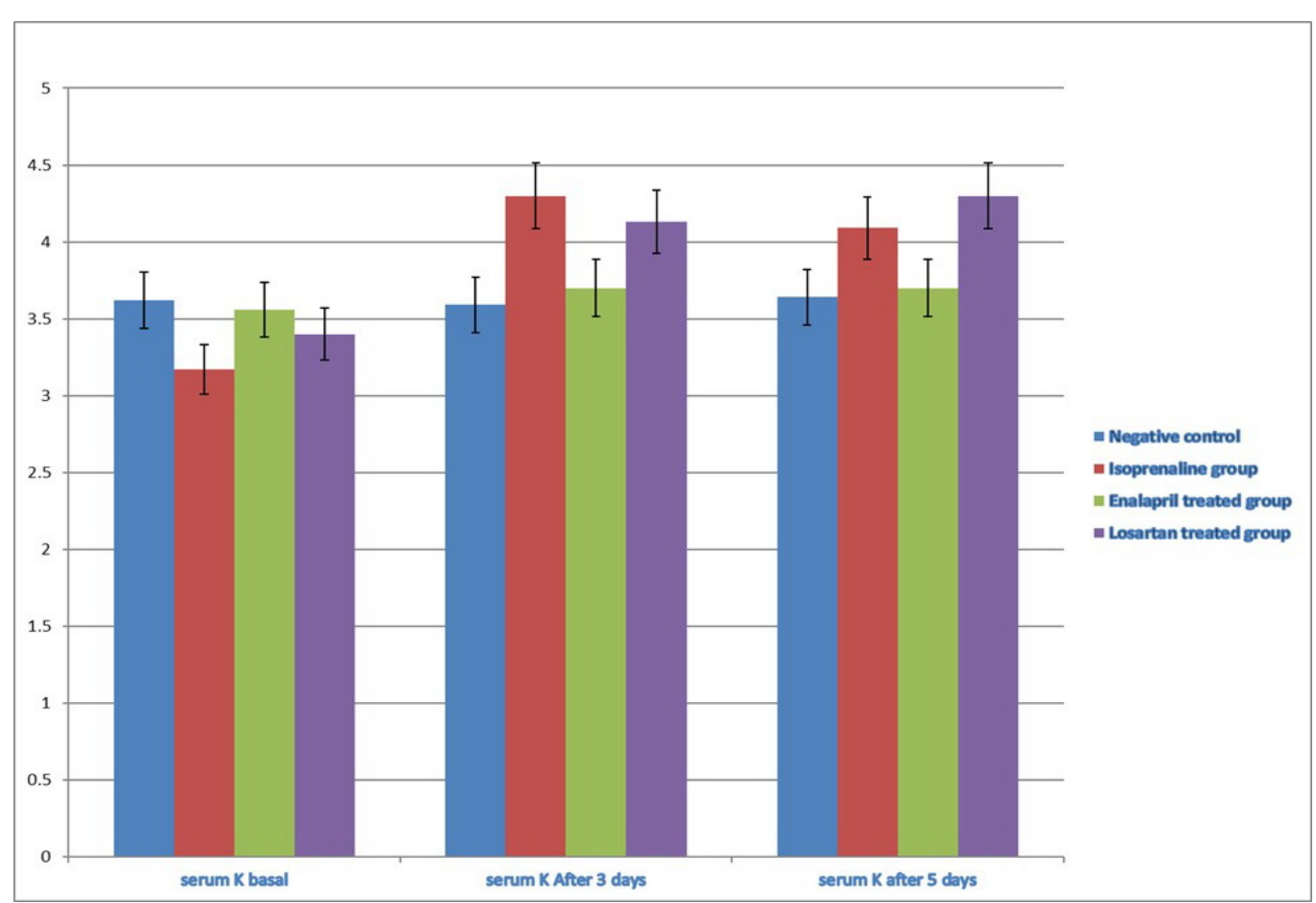

Figure (4): Effect of enalapril and losartan on serum K (meq/dl). Test used: One-way ANOVA followed by Dunnet comparison test. Values expressed as means $\pm \mathrm{SEM}^{\mathrm{P} 1}$ significance vs isoprenaline group, ${ }^{\mathrm{P} 2}$ significance vs negative group.

\section{Effect of ACEIs and ARBs on myocardial} morphology in ISO-induced MI:

Table 2 demonstrated scoring of myocardial damage in most of rats from the normal control group was A, while it was C in ISO group and $\mathrm{B}$ in both Enalapril and losartan pretreated groups. As regard histopathological examination, Figure 5B \& $\mathbf{C}$ showed that administration of ISO resulted in MI in the rats leading to an infarcted area with edema of the interstitium together with excessive inflammatory cells infiltration and myofibrillary degeneration. Meanwhile, cardiac muscle obtained from enalapril and losartan treated rats showed mild to moderate necrosis of the myocardium with edema of the interstitium Figure 5D \& E.

Table (2): The number of rats with different scores of myocardial damages in different groups

\begin{tabular}{|l|c|c|c|}
\hline & Score A & Score B & Score C \\
\hline Normal group & 5 & 1 & 0 \\
\hline ISO group & 0 & 2 & 4 \\
\hline Enalapril treated group & 1 & 3 & 2 \\
\hline Losartan treated group & 1 & 3 & 2 \\
\hline
\end{tabular}

there was significant improvement of Bcl2 of both

Figure 6A showed the relative expression of $\mathrm{Bcl} 2$ in the myocardium of all experimental rats by RT-PCR. Myocardial expression of ISO group showed significant decrease $(\mathrm{p}<0.001)$ of $\mathrm{Bcl} 2$ when compared with normal group. However, treated groups either enalapril $(\mathrm{p}<0.005)$ and losartan ( $\mathrm{p}<0.001)$ when compared to ISO group.

The myocardial expression of HO-1 and Nrf2 in all experimental rats was displayed in Figure 6B and 6C respectively. The expression of 
Nrf2 and HO-1 were significantly upregulated $(<0.001)$ in rats induced with ISO when compared with control negative group. On comparison with
ISO-induced, the expression of Nrf2 and HO-1 was furthermore upregulated $(<0.001)$ in rats pretreated with enalapril and losartan.

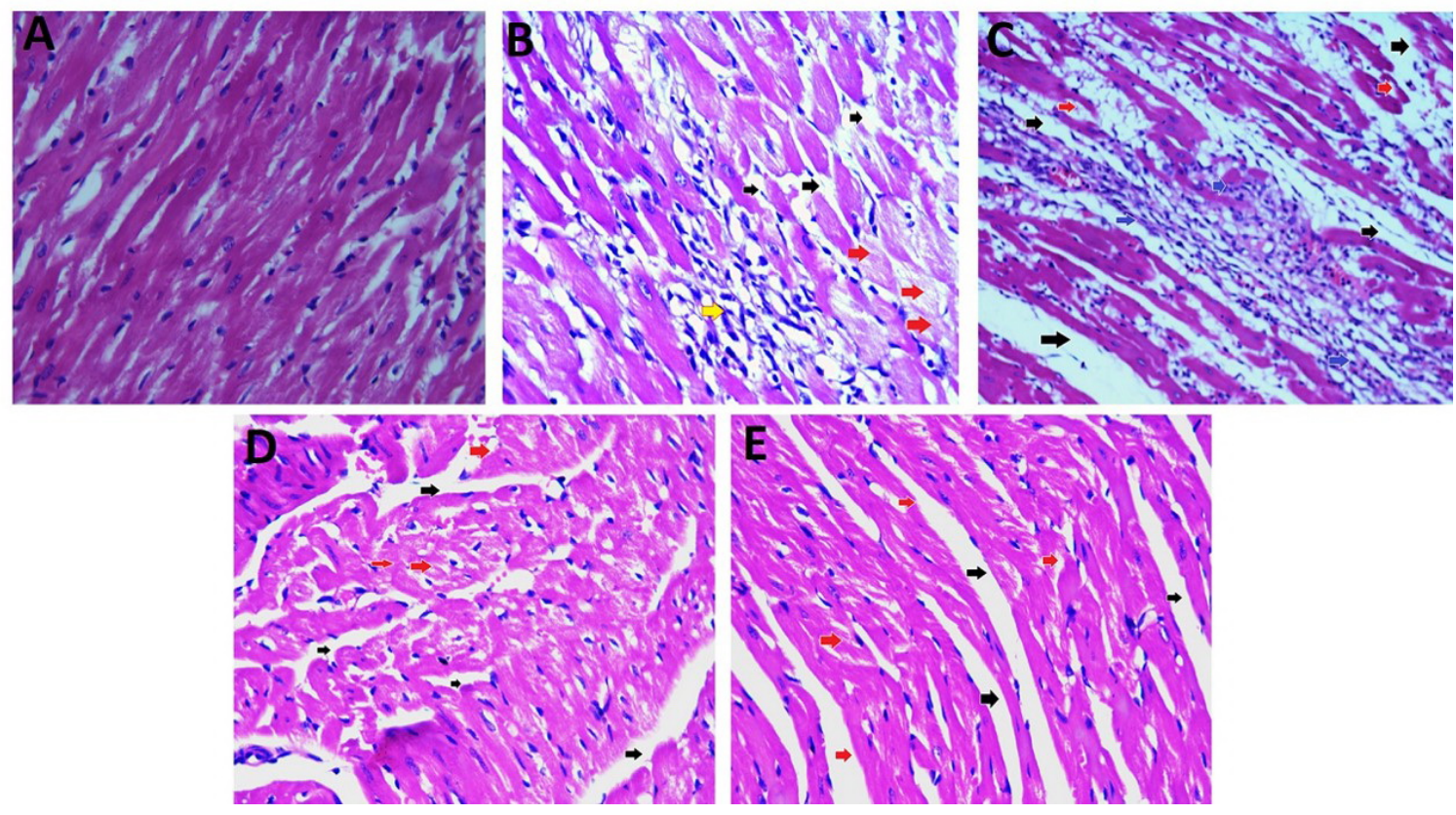

Figure (5): Histopathological examination of heart specimens. A) control negative group showing normal structure of cardiac muscle. B) isoprenaline group showing infarction area with edema of the interstitium (black arrows), marked myofibrillary degeneration (red arrows) and marked neutrophil infiltration (blue arrows). C) isoprenaline group showing infarction area with edema of the interstitium (black arrows), marked myofibrillary degeneration (red arrows) and marked neutrophil infiltration (blue arrows). D) Enalpril treated rats showing interstitial edema (black arrows) and little myofibrillary degeneration (red arrows). E) Losartan treated rats showing interstitial edema (black arrows) and little myofibrillary degeneration (red arrows) (H\&E, 400X)
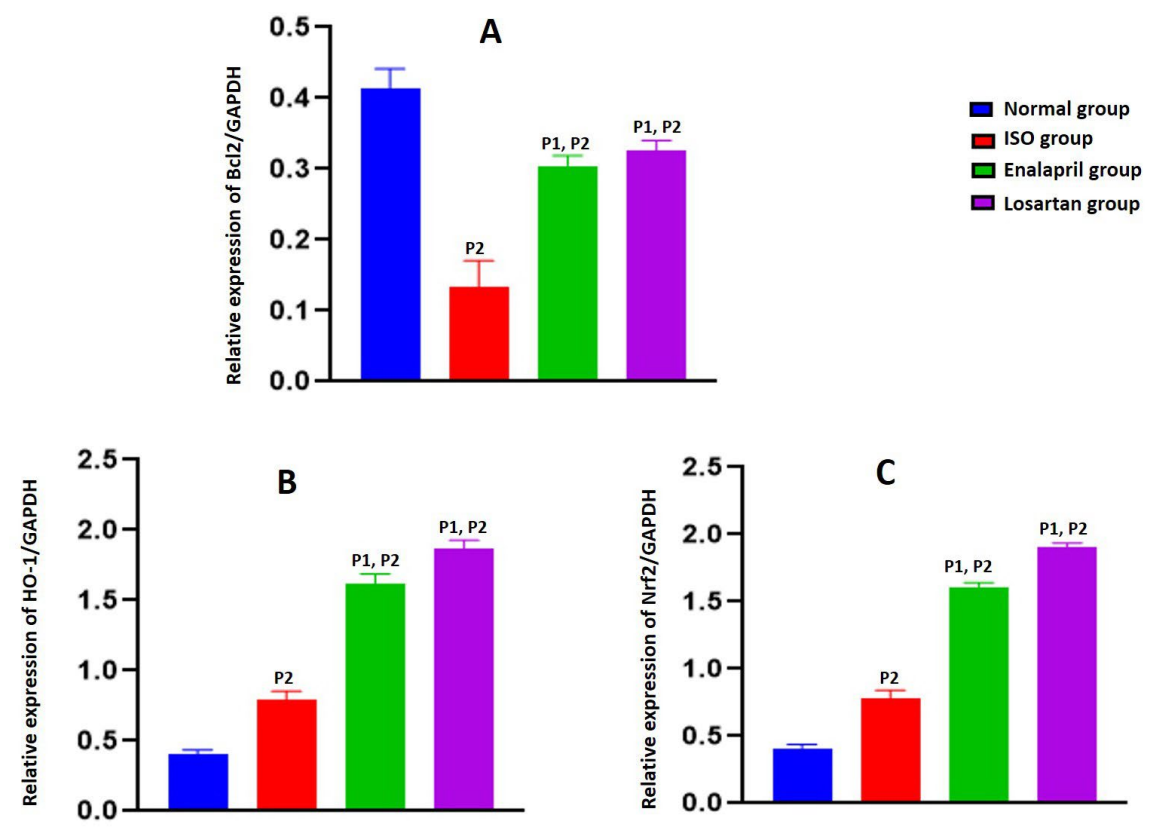

Figure (6): Effect of enalapril and losartan on myocardial relative expression of A) Bcl2, B) HO-1 and C) Nrf2. Test used: One-way ANOVA followed by Dunnet comparison test. Values expressed as means \pm SEM. ${ }^{\mathrm{P} 1}$ significance vs isoprenaline group, ${ }^{\mathrm{P} 2}$ significance vs negative group. 


\section{Discussion}

The renin angiotensin system (RAS) adjusts comprehensive sets of functions in different organs like the circulatory system and kidneys. It plays an important role in regulating extracellular volume, arterial blood pressure and tissue perfusion. Moreover, RAS improves the dynamic control of vascular function during health and disease (14).

Angiotensinogen is a protein synthesized by liver and catalyzed by renin to form angiotensin I (Ang I) that was converted into Ang II by the angiotensin converting enzyme (ACE). Also, ACE2 can hydrolyzes Ang I into Ang-(1-9) and Ang II into Ang-(1-7) and endopeptidases metabolize Ang I into the Ang-(1-7) (15). MI upregulates all components of cardiac RAS and activates an order of cellular and molecular events that initiated by a robust inflammatory response. Prolonged inflammation can impair repair of cardiac muscle and lead to heart failure $(16,17)$. Ang II triggers numerous intracellular signaling pathways which involved in inflammation, fibrogenesis, oxidative stress and cell death. In addition, Ang II controls the expression of many bioactive molecules, which responsible for pathological cardiac remodeling and impairing cardiac function in experimental animals and patients (18). Post-MI ventricular remodeling is structural and functional changes in the heart due to injury of myocardium. This process of remodeling is induced by activation of neuroendocrine system, myocardial stretch and the activation of the local tissue RAS (19). Therefore, continuous elevation of Ang II leads to progressive and irreversible cardiac remodeling (20).
Many experimental and clinical data indicate an essential role of the RAS in the setting of acute MI, at the acute phase of MI, there is a vigorous elevation in the level of Ang II and its receptor expression in both circulation and/or infarct tissues $(21,22)$. Ang II acts through type 1 (AT1) and type 2 (AT2) receptors. Both AT1 and AT2 receptors are universally expressed in the heart and AT1 mediates most of the pathophysiological actions of Ang II. Through its action on these receptors; Ang II is able to activate several intracellular signaling pathways that lead to inflammation, oxidative stress, fibrogenesis, cell death and differentiation and regulates gene expression of a widespread range of bioactive elements, eventually leading to pathological cardiac remodeling (23).

Therefore, the aim of the present study was to evaluate the effect of RAS on rats with ISO-induced MI by comparing pretreatment with ACEs enalapril (50mg/kg, body weight) and ARBs losartan (10mg/kg, body weight). Furthermore, we hypothesized that $\mathrm{Nrf} / \mathrm{HO}-1$ signaling pathway induction by enalapril and losartan could improve the cardiac functions in ISO-induced MI

Myocardial necrosis is the hallmark of acute MI that lead to leak of creatine kinase (CK)$\mathrm{MB}$ and lactate dehydrogenase (LDH) that are used as biochemical biomarkers in diagnosis (24). During this study, an increase in serum level of cardiac enzymes reflects the alterations in plasma membrane permeability as a response to $\beta$ adrenergic stimulation which induced by administration of ISO. In contrary, rats pretreated with enalapril and losartan showed marked reductions of these cardiac enzymes that appears 
on third day and continues along fifth day without a significant alteration of $\mathrm{K}$ level.

Endogenous antioxidants (GSH and catalase) and lipid peroxidation product (MDA) were measured to confirm the myocardial oxidative stress after administration of ISO. Isoproterenol produce significant decrease in the level of GSH and catalase accompanied with significant elevation of MDA when compared with control rats. This finding explained by ISO produce quinones which react with oxygen to generate superoxide anions and $\mathrm{H}_{2} \mathrm{O}_{2}$, which have damaging effects in cells (25). ISO induced oxidative stress can be characterized by reduction of myocardial catalase and GSH along with a rise in myocardial MDA level. Our results demonstrate that pretreatment with ACEs and ARBs have antioxidative effects in ISO-induced MI; enalapril and losartan prevented the elevation in MDA and decrease in the level of GSH and catalase. These results are in agreement with Shree et al. (26) that report a significant ameliorating effect of losartan on variety of parameters like glutathione, glutathione peroxidase, superoxide dismutase, and catalase and inhibitory effect on lipid peroxidant malondialdehyde. Oxidative stress plays an important role in development and progression of MI, this indicates that both enalapril and losartan have antioxidant effect against ISO-induced MI.

ISO-induced myocardial necrosis is also confirmed by histopathological findings. ISOtreated heart showed the features of severe inflammation, the inflammatory cells infiltration between the myocardial fibers with interstitial edema and areas of myocytolysis. These all changes were previously reported in various ISOinduced MI models $(27,28)$. However, cardiac muscle of rats pretreated with enalapril and losartan showed mild to moderate necrosis of the myocardium with edema of the interstitium.

Multiple evidence found that cell apoptosis is important in the pathogenesis and in remodeling of MI. several researches have investigated the apoptotic pathway in ISO-induced MI $(29,30)$. The results show that administration of ISO in rats resulted in upregulation of the expressions of myocardial pro-apoptotic signaling proteins, while the expression of anti-apoptotic Bcl-2 undergo down-regulation. In consistent with previous observations our results showed significant decrease in the level of $\mathrm{Bcl} 2$ in ISO treated rats when compared with control negative rats. Bcl-2 is an anti-apoptotic member of the Bcl2 family, by scavenging oxygen-free radicals inside the cells and repressing the release of cytochrome $\mathrm{C}$ into the cytoplasm, which play important roles in the regulation of the apoptotic pathway (31). Our results suggested that the pretreatment with enalapril and losartan, the level of Bcl-2 increased in the myocardial tissues of MI rats compared with the ISO group.

Nrf2 is a nuclear transcriptional factor which has an important role in the regulation of redox homeostasis by increase expression of antioxidant and detoxifying enzyme. Under physiological conditions, the Nrf2 form a complex with its negative regulator-Kelch-like ECM associated protein (Keap1) as an inactive form (Nrf2-Keap1 complex reside in the cytoplasm) and during stimulation this complex degraded by the proteasome (32). Under pathological condition or oxidative stress such as induced by ISO, the Nrf2 broke down from the Nrf2-Keap1 complex to released-active form and translocate from 
cytoplasm to nucleus and bound with antioxidant response element (ARE) regions and subsequently upregulate the expression of antioxidant and detoxifying enzyme genes to protect the cells from further damage (33). Previous reports demonstrated that ISO can induce oxidative stress accompanied by modulation of $\mathrm{Nrf} 2 / \mathrm{HO}-1$ signaling pathway to protect myocardium by enhancing anti-oxidant activity $(9,10)$. In agreement with these previous findings, our results showed an increase in the expression of $\mathrm{Nrf} 2$ observed in rats induced with ISO when compared with normal rats as a defense mechanism to lower the oxidative stress. Hence, in the current work, rats pre-treated with enalapril and losartan before ISO-induced MI showed furthermore expression of Nrf2 was observed in the rats pretreated with enalapril and losartan when compared with ISO group.

HO-1 has cytoprotective properties such as anti-oxidative, anti-apoptotic and antiinflammatory effects (34). It has been confirmed that increase expression of HO-1 in animals reduced infarct size, ventricular remodeling, enhanced endothelial function, induced angiogenesis and restored cardiac metabolism (35). Our study demonstrated that ISO induce significant elevation in the expression of HO-1, this effect might be due to oxidative stress induced by ISO. In line with this hypothesis, Hu et al.(36) found that ISO could be induced injury in multiple organs and cells in response to a variety of stimuli, such as oxidative stress and myocardial ischemia/reperfusion ( $/ / \mathrm{R})$. Also, activation of $\beta 1$ adrenoreceptors by ISO causes significant increase in cyclic adenosine monophosphate (cAMP), which in turn activates protein kinase A (37), and stimulates phosphoinositide 3-kinase (PI3K) and p38 mitogen activated protein kinase (MAPK) activity. Activated PI3K and p38MAPK make the Nrf2 to move to the nucleus, where binding of Nrf2 to the ARE promoter site, leads to the upregulation of HO-1 gene expression (38). We found that the expression of $\mathrm{HO}-1$ was furthermore upregulated in rats pretreated with enalapril and losartan when compared with ISO group. Our results are in correspondence with the outcome of Kim et al. (39) as well as Wang et al. (40), they both demonstrated that pretreatment with losartan enhance the expression of Nrf2 and HO-1 levels in chronic renal diseases and cavernous nerve injury in rats. Therefore, we demonstrated that enalapril and losartan can modulate the Nrf2/HO-1 signaling pathway and thus increase the expression of antioxidant thereby endorsing its cardioprotective activity.

\section{Conclusion}

Our study provides evidence that ACEIs (Enalapril) and ARBs (Losartan) ameliorates oxidative stress, inhibit apoptosis and improve cardiac dysfunction in MI by regulating bioactive markers like HO-1 and Nrf2. It also reduces cardiac enzymes and improves blood pressure without alteration of serum level of $\mathrm{K}$ on short term therapy.

Acknowledgements: The Physiology Department, Faculty of Medicine, Mansoura University acknowledged for contribution in the experimental part of the present study.

Author contribution: All authors have accepted responsibility for the entire content of this manuscript and approved its submission. 
Conflict of interest: The authors declare that there References

1. Boarescu P-M, Chirilă I, Bulboacă $\mathbf{A E}$, Bocșan IC, Pop RM, Gheban D, et al. Effects of Curcumin Nanoparticles in Isoproterenol-Induced Myocardial Infarction. Oxid Med Cell Longev. 2019;2019:7847142.

2. Yang K-C, Kyle JW, Makielski JC, Dudley SCJ. Mechanisms of sudden cardiac death: oxidants and metabolism. Circ Res. 2015 Jun;116(12):1937-55.

3. Basile J. New therapeutic options in patients prone to hypertension: a focus on direct Renin inhibition and aldosterone blockade. Am J Med Sci. 2009 Jun;337(6):438-44.

4. Zhong J-C, Ye J-Y, Jin H-Y, Yu X, Yu H-M, Zhu D-L, et al. Telmisartan attenuates aortic hypertrophy in hypertensive rats by the modulation of ACE2 and profilin-1 expression. Regul Pept. 2011 Jan;166(1-3):90-7.

5. Hill-Kapturczak N, Chang S-H, Agarwal

A. Heme oxygenase and the kidney. DNA Cell Biol. 2002 Apr;21(4):307-21.

6. Kirkby KA, Adin CA. Products of heme oxygenase and their potential therapeutic applications. Am J Physiol Renal Physiol. 2006 Mar;290(3):F563-71.

7. Zhu H, Itoh K, Yamamoto M, Zweier JL, Li Y. Role of Nrf2 signaling in regulation of antioxidants and phase 2 enzymes in cardiac fibroblasts: protection against reactive oxygen and nitrogen species-induced cell injury. FEBS Lett. 2005 Jun;579(14):3029-36. is no conflict of interest.

8. Purdom-Dickinson SE, Lin Y, Dedek M, Morrissy S, Johnson J, Chen QM. Induction of antioxidant and detoxification response by oxidants in cardiomyocytes: evidence from gene expression profiling and activation of Nrf2 transcription factor. J Mol Cell Cardiol. 2007 Jan;42(1):159-76.

9. Sahu BD, Kuncha M, Rachamalla SS, Sistla R. Lagerstroemia speciosa L. attenuates apoptosis in isoproterenolinduced cardiotoxic mice by inhibiting oxidative stress: possible role of Nrf2/HO1. Cardiovasc Toxicol. 2015 Jan;15(1):10 22.

10. Li Y, Feng J, Mo Y, Liu H, Yang B. Concordance between cardio-protective effect on isoproterenol-induced acute myocardial ischemia and phenolic content of different extracts of Curcuma aromatica. Pharm Biol. 2016 Dec;54(12):3226-31.

11. Sathish $\mathbf{V}$, Ebenezar KK, Devaki $\mathbf{T}$. Synergistic effect of Nicorandil and Amlodipine on tissue defense system during experimental myocardial infarction in rats. Mol Cell Biochem. 2003 Jan;243(12):133-8.

12. Yang $\mathbf{Y}$, Zhang $\mathbf{P}$, Song $\mathbf{L}$, Ruan $\mathbf{Y}, \mathbf{X u}$ $\mathbf{X}, \mathbf{L i} \mathbf{Y}$, et al. Comparison of three doses of enalapril in preventing left ventricular remodeling after acute myocardial infarction in the rat. Chin Med J (Engl). 2002 Mar;115(3):347-51.

13. Kaneko K, Susic D, Nunez E, Frohlich ED. Losartan reduces cardiac mass and improves coronary flow reserve in the 
spontaneously hypertensive rat. J

Hypertens. 1996 May;14(5):645-53.

14. Heras MM, Rodríguez C, González JFN. The Renin-Angiotensin-Aldosterone System in Renal and Cardiovascular Disease and the Effects of its Pharmacological Blockade ReninAngiotensin-Aldosterone System: A 2011 overview. 2012;3(1):1-24.

15. Patel VB, Clarke N, Wang Z, Fan D, Parajuli N, Basu R, et al. Angiotensin II induced proteolytic cleavage of myocardial ACE2 is mediated by TACE/ADAM-17: a positive feedback mechanism in the RAS. $\mathrm{J}$ Mol Cell Cardiol. 2014 Jan;66:167-76.

16. Sun Y. Intracardiac renin-angiotensin system and myocardial repair/remodeling following infarction. J Mol Cell Cardiol. 2010 Mar;48(3):483-9.

17. Reindl M, Reinstadler SJ, Feistritzer HJ, Mayr A, Klug G, Marschang P, et al. Acute myocardial infarction as a manifestation of systemic vasculitis. Wien Klin Wochenschr. 2016 Nov;128(2122):841-3.

18. Wong ZW, Thanikachalam PV, Ramamurthy S. Molecular understanding of the protective role of natural products on isoproterenol-induced myocardial infarction: A review. Biomed Pharmacother. 2017 Oct;94:1145-66.

19. Talman V, Ruskoaho H. Cardiac fibrosis in myocardial infarction-from repair and remodeling to regeneration. Cell Tissue Res. 2016 Sep;365(3):563-81.

20. Bader M. Role of the local reninangiotensin system in cardiac damage: a minireview focussing on transgenic animal models. J Mol Cell Cardiol. 2002 Nov;34(11):1455-62.

21. González GE, Seropian IM, Krieger ML, Palleiro J, Lopez Verrilli MA, Gironacci MM, et al. Effect of early versus late AT(1) receptor blockade with losartan on postmyocardial infarction ventricular remodeling in rabbits. Am J Physiol Heart Circ Physiol. 2009 Jul;297(1):H375-86.

22. Oyamada S, Bianchi C, Takai S, Robich MP, Clements RT, Chu L, et al. Impact of acute myocardial ischemia reperfusion on the tissue and blood-borne reninangiotensin system. Basic Res Cardiol. 2010 Jul;105(4):513-22.

23. Kumar G. Anand. Review Article Biomarkers in Acute Myocardial Infarction. 2018;8(3):137-41.

24. Chen Y, Tao Y, Zhang L, Xu W, Zhou X. Diagnostic and prognostic value of biomarkers in acute myocardial infarction. Postgrad Med J. 2019 Apr;95(1122):210-6.

25. Rathore N, Kale M, John S, Bhatnagar D. Lipid peroxidation and antioxidant enzymes in isoproterenol induced oxidative stress in rat erythrocytes. Indian J Physiol Pharmacol. 2000 Apr;44(2):161-6.

26. Shree J, Choudhary R, Bodakhe SH. Losartan delays the progression of streptozotocin-induced diabetic cataracts in albino rats. J Biochem Mol Toxicol. 2019 Aug;33(8):e22342.

27. Banerjee SK, Sood S, Dinda AK, Das TK, Maulik SK. Chronic oral administration of raw garlic protects against isoproterenol-induced myocardial 
necrosis in rat. Comp Biochem Physiol C Toxicol Pharmacol. 2003 Dec;136(4):37786.

28. Thippeswamy BS, Thakker SP, Tubachi S, Kalyani GA, Netra MK, Patil U, et al. Cardioprotective effect of Cucumis trigonus Roxb on isoproterenol-induced myocardial infarction in rat. Am J Pharmacol Toxicol. 2009;4(2):29-37.

29. Radhiga T, Rajamanickam C, Sundaresan A, Ezhumalai M, Pugalendi KV. Effect of ursolic acid treatment on apoptosis and DNA damage in isoproterenol-induced myocardial infarction. Biochimie. 2012 May;94(5):1135-42.

30. Othman AI, Elkomy MM, El-Missiry MA, Dardor M. Epigallocatechin-3-gallate prevents cardiac apoptosis by modulating the intrinsic apoptotic pathway in isoproterenol-induced myocardial infarction. Eur J Pharmacol. 2017 Jan;794:27-36.

31. Chang JW, Hwang HS, Kim YS, Kim HJ, Shin YS, Jittreetat T, et al. Protective effect of Artemisia asiatica (Pamp.) Nakai ex Kitam ethanol extract against cisplatininduced apoptosis of human $\mathrm{HaCaT}$ keratinocytes: Involvement of NF-kappa Band Bcl-2-controlled mitochondrial signaling. Phytomedicine. 2015 Jun;22(6):679-88.

32. Lee J-W, Bae CJ, Choi Y-J, Kim S-I, Kwon Y-S, Lee HJ, et al. 3,4,5trihydroxycinnamic acid inhibits lipopolysaccharide (LPS)-induced inflammation by Nrf2 activation in vitro and improves survival of mice in LPSinduced endotoxemia model in vivo. Mol Cell Biochem. 2014 May;390(1-2):143-53.

33. Cheng L, Jin Z, Zhao R, Ren K, Deng C, Yu S. Resveratrol attenuates inflammation and oxidative stress induced by myocardial ischemia-reperfusion injury: role of Nrf2/ARE pathway. Int J Clin Exp Med. 2015;8(7):10420-8.

34. Ryter SW, Alam J, Choi AMK. Heme oxygenase-1/carbon monoxide: from basic science to therapeutic applications. Physiol Rev. 2006 Apr;86(2):583-650.

35. Kusmic C, Barsanti C, Matteucci M, Vesentini N, Pelosi G, Abraham NG, et al. Up-regulation of heme oxygenase-1 after infarct initiation reduces mortality, infarct size and left ventricular remodeling: experimental evidence and proof of concept. J Transl Med. 2014 Apr;12:89.

36. Hu X, Fu W, Jiang H. HMGB1: a potential therapeutic target for myocardial ischemia and reperfusion injury. Vol. 155, International journal of cardiology. Netherlands; 2012. p. 489.

37. Sun J, Kim SJ, Park MK, Kim HJ, Tsoy I, Kang YJ, et al. Selective activation of adrenergic betal receptors induces heme oxygenase 1 production in RAW264.7 cells. FEBS Lett. 2005 Oct;579(25):5494500.

38. Ha YM, Ham SA, Kim YM, Lee YS, Kim HJ, Seo HG, et al. $\beta_{1}$-adrenergic receptormediated HO-1 induction, via PI3K and p38 MAPK, by isoproterenol in RAW 264.7 cells leads to inhibition of HMGB1 release in LPS-activated RAW 264.7 cells 
and increases in survival rate of CLPinduced septic mice. 'Biochemical Pharmacol. 2011 Oct;82(7):769-77.

39. Kim H, Baek CH, Lee RB, Chang JW, Yang WS, Lee SK. Anti-Fibrotic Effect of Losartan, an Angiotensin II Receptor Blocker, Is Mediated through Inhibition of ER Stress via Up-Regulation of SIRT1, Followed by Induction of HO-1 and
Thioredoxin. Int J Mol Sci. 2017 Jan;18(2).

40. Wang Y, Meng X-H, Zhang Q-J, Wang Y-M, Chen C, Wang Y-C, et al. Losartan improves erectile function through suppression of corporal apoptosis and oxidative stress in rats with cavernous nerve injury. Asian J Androl. 2019;21(5):452-9. 